\title{
Plan nacional de detección y tratamiento de la retinopatía del prematuro
}

\section{National plan for detection and treatment of retinopathy of prematurity}

\author{
Luis P. Orozco-Gómez $z^{1,2,3 *}$
}

${ }^{1}$ Presidente de la Sociedad Panaméricana de Retinopatía del Prematuro; ${ }^{2}$ Grupo ROP México; ${ }^{3}$ Servicio de Oftalmología, Centro Médico Nacional 20 de Noviembre. Ciudad de México, México

\section{Resumen}

Se presenta el resumen histórico del trabajo realizado por la Sociedad Mexicana de Oftalmología, el Grupo ROP México y la Secretaría de Salud para el desarrollo del plan nacional de detección y tratamiento de la retinopatía del prematuro.

Palabras clave: Plan Nacional ROP México. Prematuro. Retinopatía. Ceguera.

\section{Abstract}

We present a historical summary of the work performed by the Sociedad Mexicana de Oftalmología, the ROP Mexico Group and the Ministry of Health for the development of the national plan for detection and treatment of premature retinopathy.

Key words: National plan ROP Mexico. Prematurity. Retinopathy. Blindness.

\section{Correspondencia:}

*Luis P. Orozco-Gómez

San Francisco, 1626 - 605

Col. Del Valle

Fecha de recepción: 09-12-2018

Fecha de aceptación: 18-07-2019

C.P. 03100, Ciudad de México, México

E-mail: Iporozco@issste.gob.mx

DOI: 10.24875/RMO.M19000088
Disponible en internet: 01-11-2019 Rev Mex Oftalmol. 2019;93(6):295-302

www.rmo.com.mx (http://creativecommons.org/licenses/by-nc-nd/4.0/). 
Para entrar en contexto de lo que significa la retinopatía del recién nacido prematuro (ROP), tendríamos que entender que es una patología que solamente se presenta en aquellos niños producto de un embarazo que se ve interrumpido antes de completar el crecimiento y desarrollo normal que un bebé tiene durante las 40 semanas de embarazo.

Las causas para que un bebé nazca prematuramente son muy diversas, y tienen que ver con enfermedades maternas o con alteraciones que afectan al producto. Todos estos recién nacidos prematuros deben ser ingresados en unidades de cuidados intensivos, donde la intervención de los neonatólogos y enfermeras es crucial para que puedan desarrollarse y así lograr sobrevivir. Una vez pasado este periodo crítico, alrededor de la cuarta semana de vida, debe valorárseles el fondo de ojo para determinar el estado vascular de la retina y tomar decisiones terapéuticas para evitar la ceguera de estos pacientes por esta enfermedad.

A mediados de la década de 1980 se iniciaron algunos programas de detección de ROP, principalmente en ciudades como Guadalajara, Distrito Federal y Monterrey. Con la finalidad de evitar la ceguera en estos pacientes, estos centros iniciaron sus programas y fueron acumulando casos y experiencia en su manejo. $A$ partir del año 2000 se redoblaron los esfuerzos basándose en el Programa visión 20/20 de la Organización Mundial de la Salud (OMS), cuyo objetivo general era lograr la reducción en un $50 \%$ de los casos prevenibles de ceguera para el año 2020.

En el año 2001, el Dr. Federico Graue, siendo presidente de la Sociedad Mexicana de Oftalmología, convocó a todos los médicos de hospitales generales y oftalmológicos que teníamos relación con pacientes recién nacidos prematuros a la reunión organizada por el International Council of Ophthalmology para conocer la realidad del diagnóstico y tratamiento de la ROP en nuestro país. Para dicha reunión, fueron invitadas como conferencistas la Dra. Clare E. Gilbert, del Eye Health International Centre, la Dra. Andrea Zin, de la Fundación Oswaldo Cruz, Rio de Janeiro (FIOCRUZ), y la Dra. Luz Gordillo Robles, del instituto Damos de Lima, Perú, con la finalidad de conocer la realidad mexicana para poder dimensionar el problema, ya que no había información en la literatura respecto a este tema, y solo el banco mundial tenía un estimado del número de ciegos anuales que por esta patología teníamos en México.

En dicha reunión, solamente en tres hospitales se tenía un plan formal de tamizaje de ROP. El Centro Médico Nacional (CMN) 20 de Noviembre del Instituto de Seguridad y Servicios Sociales de los Trabajadores del Estado (ISSSTE), el Hospital de Pediatría del Centro Médico Nacional Siglo XXI del Instituto Mexicano del Seguro Social (IMSS) y el Hospital Civil de Guadalajara.

Desde ese año que se realizó la primera reunión nacional de trabajo sobre ROP con el apoyo e intervención de diversos grupos públicos, sociales y privados, como la Sociedad Mexicana de Oftalmología, la Asociación Mexicana de Retina, diversas ONG, como CBM (Christoffel Blinden Mission) y la Red Nacional para la Prevención a la Discapacidad, entre otras, la industria farmacéutica, como Laboratorios Grin, Laboratorios Allergan, entre otros, la Secretaría de Salud a través del Centro Nacional de Equidad de Género y Salud Reproductiva, la Sociedad Iberoamericana de Neonatología y la Agencia Internacional para la Prevención de la Ceguera, asociaciones médicas pediátricas, como la Asociación Mexicana de Pediatría, la Federación Nacional de Neonatología de México, y de asociaciones de enfermería, como la Asociación Mexicana de Enfermeras Neonatales, se ha logrado que cada día haya más programas de detección y tratamiento de la ROP en nuestro país y, aunque su incremento ha sido muy importante, aún falta mucho por hacer.

El 4 de marzo de 2004 apareció publicado en el Diario Oficial de la Federación, la creación del Consejo Nacional para la Prevención y el tratamiento de las Enfermedades Visuales como el órgano consultivo e instancia permanente de coordinación y concertación de las acciones de los sectores público, social y privado en materia de investigación, prevención, diagnóstico y tratamiento integral de las enfermedades visuales detectadas en la población de la República Mexicana.

En el año de 2005 se conformó el Grupo ROP México con la valiosa intervención de distintos especialistas, principalmente oftalmólogos generales, oftalmólogos pediatras, retinólogos, pediatras, neonatólogos y enfermeras neonatales, entre otros, y con el apoyo de la industria farmacéutica, ONG y organismos gubernamentales. Se realizaron distintas pláticas de difusión sobre el tema en distintos escenarios, como hospitales estatales con unidades neonatales, congresos médicos de pediatría, oftalmología, neonatología y de enfermería, tanto a nivel nacional como internacional. En 5 años se realizaron más de 50 intervenciones de este tipo, en las que también se intentó sembrar la semilla de la integración del tema de la ROP en las currículos de medicina general y de las distintas especialidades médicas involucradas en el manejo de ROP, así como 
incluir en la difusión de este padecimiento a los padres de niños prematuros. También se distribuyeron trípticos y pósters informativos sobre el tema en hospitales obstétricos y unidades neonatales.

En el año 2005, un grupo de médicos oftalmólogos y neonatólogos, así como de enfermeras neonatólogas, participaron en la conformación de las «Pautas para el examen, detección y tratamiento de la retinopatía del prematuro en países de Latinoamérica", las cuales se distribuyeron en Latinoamérica y en nuestro país. Fue el primer instrumento de difusión medico masivo referente al tema de ROP.

El Grupo ROP México se dio a la tarea de realizar reuniones anuales para recabar información sobre la ROP en México, y gracias a esta información se ha podido tener una pequeña muestra sobre el tema y entender su importancia y relevancia como problema de salud pública en nuestro país. En el año 2007 realizó una encuesta en escuelas para débiles visuales y ciegos en tres estados del país con la finalidad de identificar la causa primaria de esta discapacidad. En Michoacán se encontró que en las dos escuelas de este tipo del estado, la ROP contribuía con el 40 y $60 \%$ en cada una de ellas como causa de ceguera. En el estado de Morelos, en un centro se encontró que la ROP y las distrofias tapeto-retinianas eran las causas más frecuentes de ceguera y ocupaban cada uno de estos dos grupos de patologías el $14 \%$ de la población escolar con ceguera. En el Distrito Federal, en el Centro de Rehabilitación de Ciegos y Débiles Visuales del Instituto de Oftalmología, se encontró que el 30\% de los casos de ceguera en menores de 18 años de edad eran por prematuridad.

En virtud de la afiliación de nuestro país a través de la Secretaría de Salud Federal al Programa visión 20/20 de la OMS, en el año 2005, el Centro Nacional de Equidad de Género y Salud Reproductiva de la propia Secretaría de Salud realizó reuniones de trabajo con cerca de 100 participantes entre médicos oftalmólogos, retinólogos, pediatras, neonatólogos, enfermeras neonatólogas, con representantes de la gran mayoría de los estados de nuestro país. Se publicó en octubre de 2007 «Lineamiento Técnico del Manejo de la Retinopatía del Recién Nacido Prematuro" (ISBN 978-970-721-441-5), el cual se distribuye a través de la Secretaría de Salud a todos los hospitales del sistema de salud de nuestro país y colabora para indicar la necesidad de iniciar programas de detección y tratamiento de la ROP en centros hospitalarios de atención a prematuros. Este lineamiento técnico tuvo una segunda reimpresión en octubre de 2008. Aun cuando

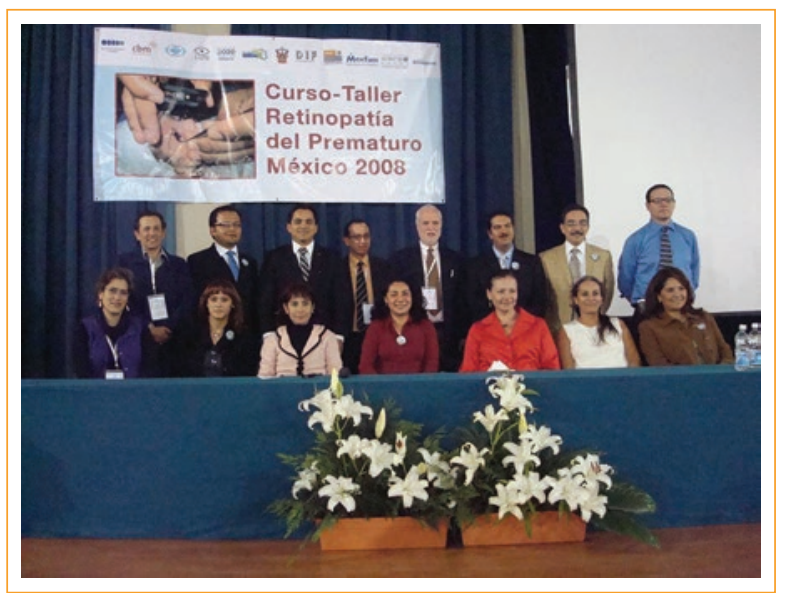

Figura 1. Primer curso taller de ROP en la ciudad de Guadalajara, 2008.

estos lineamientos técnicos emitidos por la Secretaría de Salud Federal no tienen el carácter de obligatoriedad, son recomendaciones que se realizan a todo el sistema de salud nacional (Secretaría de Salud (SSA), Instituto Mexicano del Seguro Social (IMSS), Instituto de Seguridad y Servicios Sociales de los Trabajadores del Estado (ISSTE), Petróleos Mexicanos (PM), Secretaría de la Defensa Nacional (SEDENA), etc.), lo cual fue un gran avance porque son pocas las enfermedades en las que, debido a su importancia como problemas de salud pública, se realizan grupos de trabajo multidisciplinarios, como en el caso de la ROP, para emitir estos lineamientos.

Durante 2008 se realizó un curso taller en la ciudad de Guadalajara (Fig. 1) y una encuesta a nivel nacional sobre programas de detección y tratamiento de la ROP con la participación de 10 estados de la República Mexicana y un total de 122 unidades neonatales tanto públicas como privadas. Se obtuvieron los siguientes resultados: de 122 unidades neonatales, solo en 26 de ellas se realizaba algún tipo de detección y/o tratamiento de la ROP, es decir, solo en un $21 \%$ de las unidades; el tipo de tratamiento empleado para los casos graves de ROP era crioterapia en un 33\% y láser en un $67 \%$, y solo dos centros realizaban cirugía intraocular para estadios IV o V de la enfermedad. Se obtenían resultados favorables, sin importar el tipo de tratamiento utilizado, en el $82 \%$ de los casos.

Durante el año 2011 y debido a la relevancia de la ROP, la Secretaría de Salud a través del Centro Nacional de Equidad de Género y Salud Reproductiva, incorporó el tema de diagnóstico y tratamiento de la ROP en las modificaciones que se llevaron a cabo en la Norma Oficial Mexicana 034 para la Prevención y 


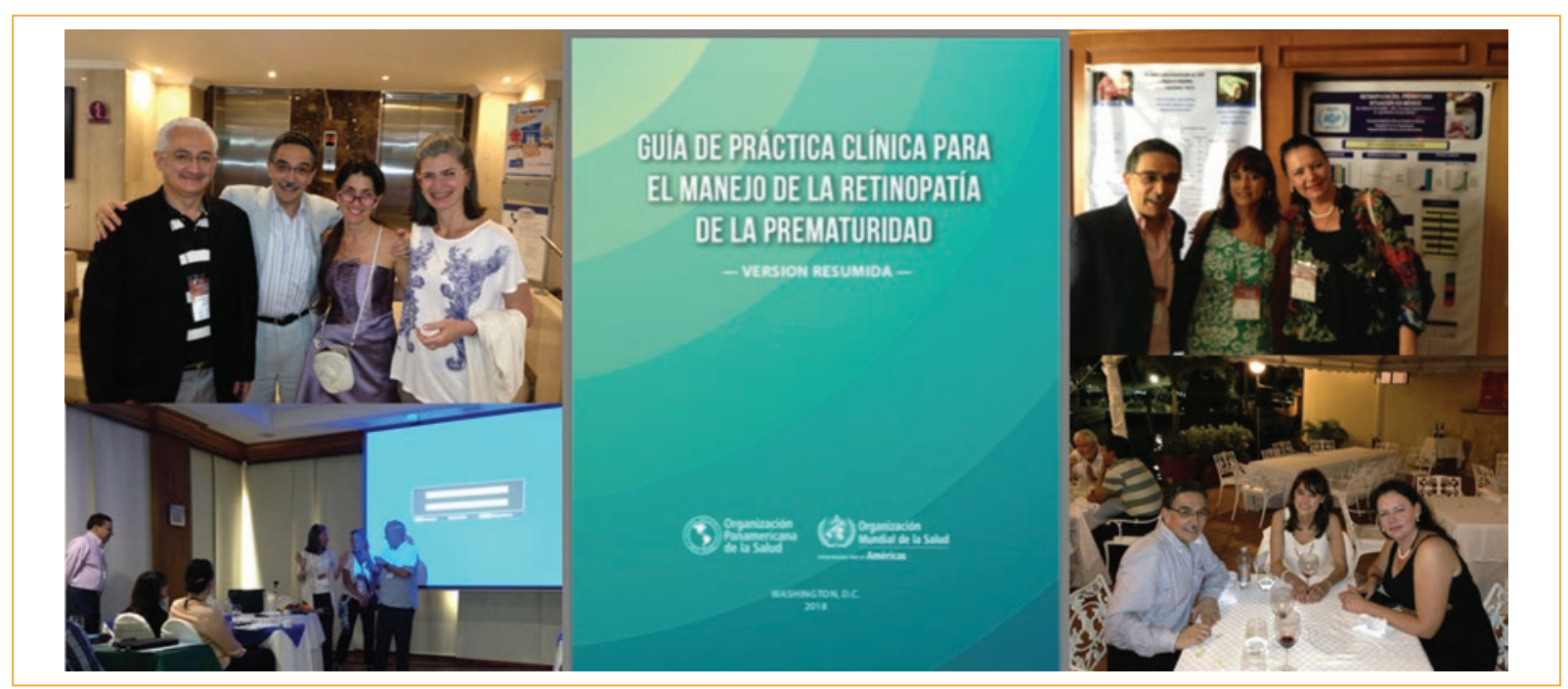

Figura 2. Participación del grupo ROP México en la guía de práctica clínica para el manejo de la retinopatía de la prematuridad.

Control de los Defectos al Nacimiento, esta NOM 034, con las correspondientes modificaciones. En el año 2012 se presentó ante el poder legislativo para su discusión y aprobación, lo cual en su momento representó un cambio radical en el manejo de la ROP en México, ya que entonces el manejo integral de la ROP en el ámbito médico, tanto público como privado, fue de observancia obligatoria con las correspondientes implicaciones médico-legales y en beneficio de los niños prematuros mexicanos.

El Grupo ROP México realizó una nueva encuesta en julio de 2011. Se obtuvo información de 14 estados de la República Mexicana, con un total de 79 unidades neonatales, públicas exclusivamente. En 42 unidades (53\%) se realizaban programas de detección de ROP y en 35 unidades (44\%) se otorgaba tratamiento de ROP. El tipo de tratamiento empleado para la ROP fue láser en el $20 \%$ de las unidades, tratamiento combinado de láser más algún antiangiogénico (ranibizumab y/o bevacizumab) en el $60 \%$ y uso de antiangiogénico como tratamiento único en el $20 \%$, lo cual da cuenta del aumento de programas de ROP en nuestro país, y el cambio que se ha realizado en pocos años en el manejo de la ROP, lo que implica consideraciones médicas, legales y éticas, por mencionar algunas, y la necesidad imperiosa de investigación en este rubro.

El 25 de enero de 2013, en el Diario Oficial de la Federación, se publicó un decreto de la Ley General de Salud, que reformaba el artículo 61, donde se señala la obligatoriedad de la revisión de la retina de todo recién nacido a la cuarta semana de nacimiento para la detección temprana de malformaciones que puedan causar ceguera y su tratamiento en todos sus grados.

En el mes de septiembre de 2013, en la ciudad de Cartagena de Indias, Colombia, fuimos convocados los oftalmólogos y neonatólogos de los países latinoamericanos para la conformación de las guías de detección y manejo de recién nacidos prematuros con ROP, fueron 3 días de intenso trabajo, que llegaron a buen fin con la publicación posterior de las guías (Figs. 2 y 3).

El 3 de junio de 2014, a solicitud de la OMS y por decreto presidencial, se creó el Consejo Nacional para la Prevención y Tratamiento de las Enfermedades Visuales, cuyo objetivo sería la prevención y eliminación de la ceguera y la discapacidad visual evitable, encabezado por la Dra. Mercedes Juan, en ese tiempo Secretaria de Salud. Se propusieron 10 estrategias, dentro de las cuales la n. ${ }^{\circ} 6$ era el tamizaje y tratamiento de la ROP.

El Comisionado Nacional del Seguro Popular, Dr. Gabriel O'Shea Cuevas, el Dr. Víctor Manuel Villagrán Muñoz, Director General Adjunto del Seguro Médico siglo XXI, la Dra. Luz Consuelo Zepeda Romero, retinóloga del Hospital Civil de Guadalajara, y el Dr. Luis Porfirio Orozco Gómez, retinólogo del Grupo ROP México, fuimos convocados para orquestar esta estrategia y crear el Plan Nacional para que en al menos un hospital acreditado de cada estado de la Secretaría de Salud donde nazcan niños fuera dotado con un equipo y los insumos necesarios para la detección y tratamiento de la ROP, ya que el 25 de enero de 2013 se había modificado el artículo 61 de la Ley General de la Salud, 


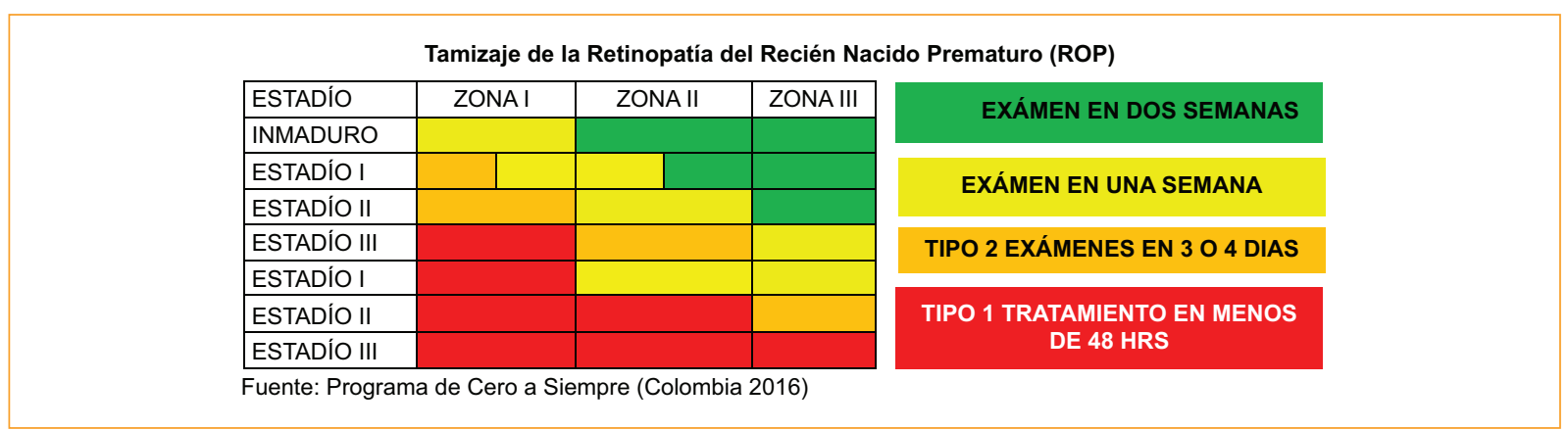

Figura 3. El grupo ROP México adoptó el algoritmo del programa de Cero a Siempre que Colombia creó en el año 2016.

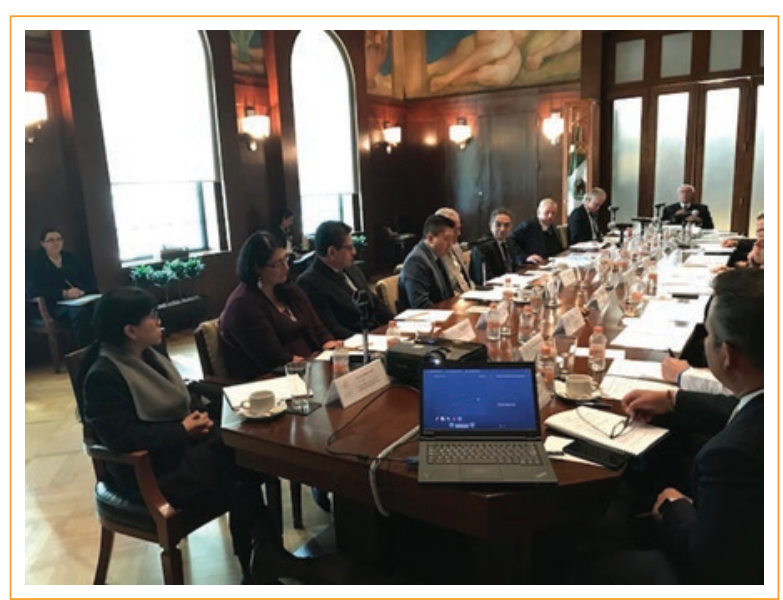

Figura 4. Reunión del consejo nacional para la prevención y tratamiento de las enfermedades visuales.

donde se había hecho obligatoria la revisión de la retina de todo recién nacido a la cuarta semana de vida y se había incluido en la cartilla nacional de salud la exploración de integridad de:

- Visual (solo por el médico) antes de los 28 días.

- La exploración de la función visual (solo por el médico) de los 6 a los 8 meses

- La toma de agudeza visual entre los 4 a los 6 años

Se realizaron múltiples reuniones en la Secretaría de Salud, las cuales se llevaron a cabo en forma ininterrumpida a pesar de que la Dra. Juan fuera sustituida por el Dr. José Narro Robles y a que el Dr. Gabriel O'Shea fuera sustituido por el Mtro. Antonio Chemor Ruiz (Fig. 4).

El Programa Seguro Médico Siglo XXI, en coordinación con el Instituto Nacional de Perinatología, han diseñado una estrategia denominada «Apoyo Vital Integral del Neonato" (AVIN), que comprende el uso de ventilación no invasiva y la aplicación racional de oxígeno regulado al prematuro comprometido. Dicha estrategia contempla la implementación de un programa de capacitación al personal de salud (pediatras, neonatólogos, enfermeras) responsable de la atención neonatal en la prevención de ROP. Como parte de la estrategia AVIN, se ha realizado la dotación de sistemas para aplicación de presión positiva continua de las vías respiratorias, con mezcladores de oxígeno y humidificadores.

Se espera lograr con todas estas acciones:

- Creación de una Red Nacional multidisciplinaria entre enfermeras, neonatólogos y oftalmólogos preocupados por el manejo correcto de los niños prematuros

- La atención oportuna del recién nacido prematuro.

- La disminución de la tasa de prevalencia anual de 500 niños ciegos por retinopatía que se tienen registrados.

- Presentar los avances médicos que se tienen en México y las posibles interrelaciones de todas las instancias para el bienestar de los recién nacidos prematuros.

Todo ello hizo necesario el establecimiento de centros regionales de tratamiento interdisciplinario para pacientes con ROP, un programa de seguimiento, así como la evaluación del impacto que permitirá demostrar la efectividad de la estrategia que finalmente habrá de contribuir a la disminución de la discapacidad visual.

A través de Programa Seguro Médico Siglo XXI, se está financiando la creación de 36 centros regionales de tratamiento interdisciplinario (uno por cada estado de la república) para pacientes con ROP, que serán operados por los servicios estatales de salud, cuyo objetivo será el de contribuir a la disminución de la discapacidad visual. En septiembre 2017 se realizó la entrega de la primera etapa (Tabla 1) de 
Tabla 1. Plan nacional ROP, primera etapa

\begin{tabular}{|c|c|c|c|c|}
\hline No & Entidad & Hospital (municipio) & Oftalmólogo & Equipo médico \\
\hline 1 & Coahuila & Hospital Universitario de Saltillo & Subrogado & Láser/Oftalmos Ind. \\
\hline 2 & Colima & Hospital Regional Universitario & Subrogado & Láser/Oftalmos Ind. \\
\hline 3 & Chiapas & $\begin{array}{l}\text { Hospital Regional Dr. Rafael Pascasio } \\
\text { Gamboa (Tuxtla) }\end{array}$ & Subrogado & Láser/Oftalmos Ind. \\
\hline 4 & Chihuahua & Hospital de la Mujer (Cd. Juárez) & Subrogado & Láser/Oftalmos Ind. \\
\hline 5 & Ciudad De México & Hospital Materno Infantil (Inguaran) & Subrogado & Láser/Oftalmos Ind. \\
\hline 6 & Chilpancingo & Hospital de la Madre y del Niño (Chilpancingo) & Subrogado & Láser/Oftalmos Ind. \\
\hline 7 & Jalisco & Hospital General de Occidente (Guadalajara) & Subrogado & Láser/Oftalmos Ind. \\
\hline 8 & Michoacán & Hospital de la Mujer (Morelia) & Subrogado & Láser/Oftalmos Ind. \\
\hline 9 & Morelos & Hospital del Niño Morelense & Subrogado & Láser/Oftalmos Ind. \\
\hline 10 & Nayarit & Hospital civil Dr. Antonio González Guevara & Subrogado & Láser/Oftalmos Ind. \\
\hline 11 & Oaxaca & $\begin{array}{l}\text { Hospital de Especialidades de la Niñez } \\
\text { Oaxaqueña (San Bartolo Coyotepec) }\end{array}$ & Subrogado & Ret-Cam/Láser \\
\hline 12 & Querétaro & $\begin{array}{l}\text { Hospital de Especialidades del Niño y la Mujer } \\
\text { Felipe Núñez Lara }\end{array}$ & Subrogado & Láser/Oftalmos Ind. \\
\hline 13 & Quintana Roo & Hospital Materno Infantil (Morelos) & Subrogado & Láser/Oftalmos Ind. \\
\hline 14 & Tabasco & $\begin{array}{l}\text { Hospital Regional de Alta Especialidad Dr. Rodolfo } \\
\text { Nieto Padrón }\end{array}$ & Subrogado & Láser/Oftalmos Ind. \\
\hline 15 & Tlaxcala & Hospital Infantil de Tlaxcala & Subrogado & Láser/Oftalmos Ind. \\
\hline 16 & Veracruz & Hospital Regional Río Blanco & Subrogado & Ret-Cam/Láser \\
\hline
\end{tabular}

A todas las unidades se las equipó con 3 indentadores esclerales tipo Flynn, tres blefaróstatos Barraquer para prematuros, lupa asférica, tres oxímetros de pulso neonatal pediátrico y un oftalmoscopio binocular indirecto.

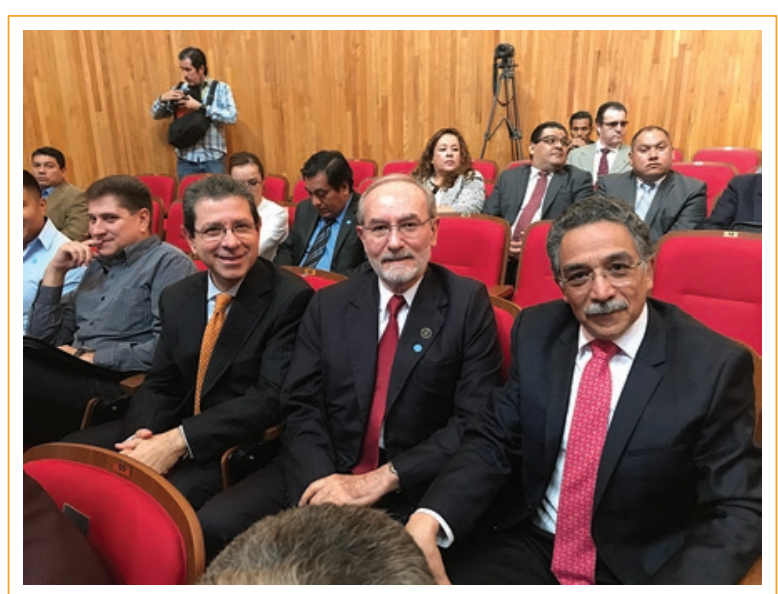

Figura 5. En la foto de izquierda a derecha: Dr. Javier Navarro, representante del Presidente de la SMO 2018; Dr. Francisco Martínez Castro, Presidente de la IAPB; y Dr. Luis Porfirio Orozco, Presidente SP-ROP.

equipamiento en 18 estados del país, a los que se equipó con una Ret-Cam y un láser de estado sólido estratégicamente por decisión de la Secretaría de Salud.

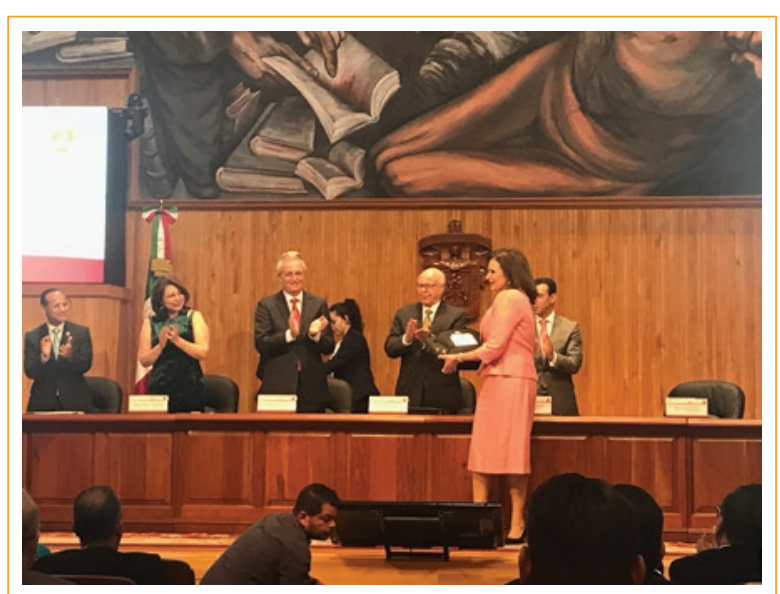

Figura 6. Dra. Consuelo Zepeda del estado de Jalisco recibe equipamiento de manos del secretario de salud, Dr. José Narro Robles.

Se inició la capacitación a médicos neonatólogos, pediatras y enfermeras en el uso adecuado de ventilación no invasiva y en la aplicación racional de oxigeno regulado al prematuro comprometido en los hospitales 
Tabla 2. Plan nacional ROP, segunda etapa

\begin{tabular}{|c|c|c|c|c|}
\hline No & Entidad & Hospital (municipio) & Oftalmólogo & Equipo médico \\
\hline 1 & Aguascalientes & $\begin{array}{l}\text { Hospital de la Mujer (Cd. Satélite } \\
\text { Morelos) }\end{array}$ & Subrogado & Láser \\
\hline 2 & Baja California & Hospital Materno Infantil (Mexicali) & Dra. Natalia I. Matti Amador & Ret-Cam/Láser \\
\hline 3 & Baja California Sur & $\begin{array}{l}\text { Hospital General con Especialidades } \\
\text { Juan María de Salvatierra (La Paz) }\end{array}$ & Subrogado & Láser/Oftalmos Ind. \\
\hline 4 & Campeche & $\begin{array}{l}\text { HG Especialidades Dr. Juan Buenfil } \\
0 \text { (Campeche) }\end{array}$ & Dra. María Mayorga Castellanos & Ret-Cam/Láser \\
\hline 5 & Ciudad de México & Hospital Infantil de México (CDMX) & Dra. Norma Citlalli Lara Molina & Ret-Cam/Láser \\
\hline 6 & Durango & $\begin{array}{l}\text { Hospital General de } \\
\text { Durango (Durango) }\end{array}$ & Dra. Tania B. Alarcón Henao & Ret-Cam/Láser \\
\hline 7 & Guanajuato & Hospital Regional de León (León) & Dr. Olivo Gómez Vargas & Ret-Cam/Láser \\
\hline 8 & Hidalgo & Hospital General Pachuca (Pachuca) & Dra. Irma Adriana González S. & Ret-Cam/Láser \\
\hline 9 & Jalisco & $\begin{array}{l}\text { Hospital Civil de } \\
\text { Guadalajara (Guadalajara) }\end{array}$ & Dra. Consuelo Zepeda Romero & Ret-Cam/Láser \\
\hline 10 & México & $\begin{array}{l}\text { Hospital Materno Perinatal Mónica } \\
\text { Pretelini (Toluca) }\end{array}$ & Dr. Hugo Tapia & Ret-Cam/Láser \\
\hline 11 & Nuevo León & $\begin{array}{l}\text { Hospital Regional Materno } \\
\text { Infantil (Guadalupe) }\end{array}$ & Dra. Andrea Rangel Padilla & Ret-Cam/Láser \\
\hline 12 & Puebla & Hospital de la Mujer (Puebla) & Dr. Luis Alberto Ramírez P. & Ret-Cam/Láser \\
\hline 13 & San Luis Potosí & $\begin{array}{l}\text { Hospital Central Dr. Ignacio Morones } \\
\text { Prieto SLP }\end{array}$ & Dra. Martha Griselda Rangel & Ret-Cam/Láser \\
\hline 14 & Sinaloa & Hospital de la Mujer (Culiacán) & Dra. Ruth Acuña Maldonado & Ret-Cam/Láser \\
\hline 15 & Sonora & $\begin{array}{l}\text { Hospital Infantil Dra. Luisa María } \\
\text { Godoy Olvera (Hermosillo) }\end{array}$ & Dr. Néstor Hugo Garrido & Ret-Cam/Láser \\
\hline 16 & Tamaulipas & $\begin{array}{l}\text { Hospital General Materno } \\
\text { Infantil (Reynosa) }\end{array}$ & Dr. Víctor González & Ret-Cam/Láser \\
\hline 17 & Yucatán & $\begin{array}{l}\text { Hospital General Agustín } \\
0 \times \text { Horan (Mérida) }\end{array}$ & Dr. Alejandro Solís Castillo & Ret-Cam/Láser \\
\hline 18 & Zacatecas & Hospital de la Mujer (Guadalupe) & Dra. Adriana Ramírez López & Ret-Cam/Láser \\
\hline
\end{tabular}

A todas las unidades se las equipó con 3 indentadores esclerales tipo Flynn, tres blefaróstatos Barraquer para prematuros, lupa asférica, tres oxímetros de pulso neonatal pediátrico y un oftalmoscopio binocular indirecto.

Materno de Celaya y del Estado de México, así como la contratación de oftalmólogos capacitados en detección de ROP, los cuales, mediante pago por evento, harán el tamizaje y tratamiento de los pacientes con ROP en estos centros.

El día 17 de septiembre del año 2018 fue un día muy importante, ya que se concluyó la entrega del equipamiento de los 33 centros de detección y manejo de pacientes con ROP. Para la ceremonia se eligió el espléndido paraninfo de la Universidad de Guadalajara, donde los fantásticos murales de José Clemente Orozco fueron testigos de tan importante ceremonia, en la cual se concretó la entrega de la segunda etapa de equipamiento (oftalmoscopios binoculares indirectos con lupa, indentador, blefarostato, oxímetro de pulso y láser de estado sólido) (Tabla 2) a los hospitales restantes de la Secretaría de Salud que cuentan con la infraestructura para recibir y tratar prematuros, concluyéndose la segunda etapa del equipamiento.

La Dra. Luz Consuelo Zepeda fue la representante de los oftalmólogos del país, pues el hospital Civil de Guadalajara fue pionero en modificación de leyes estatales para incluir la revisión de los prematuros.

Se presentó un vídeo donde se explicó la historia de la ROP y la modificación estatal de Jalisco de la ley que sirvió para finalmente en el año 2013 modificar el artículo 61 de la Ley General de Salud, donde se hizo 


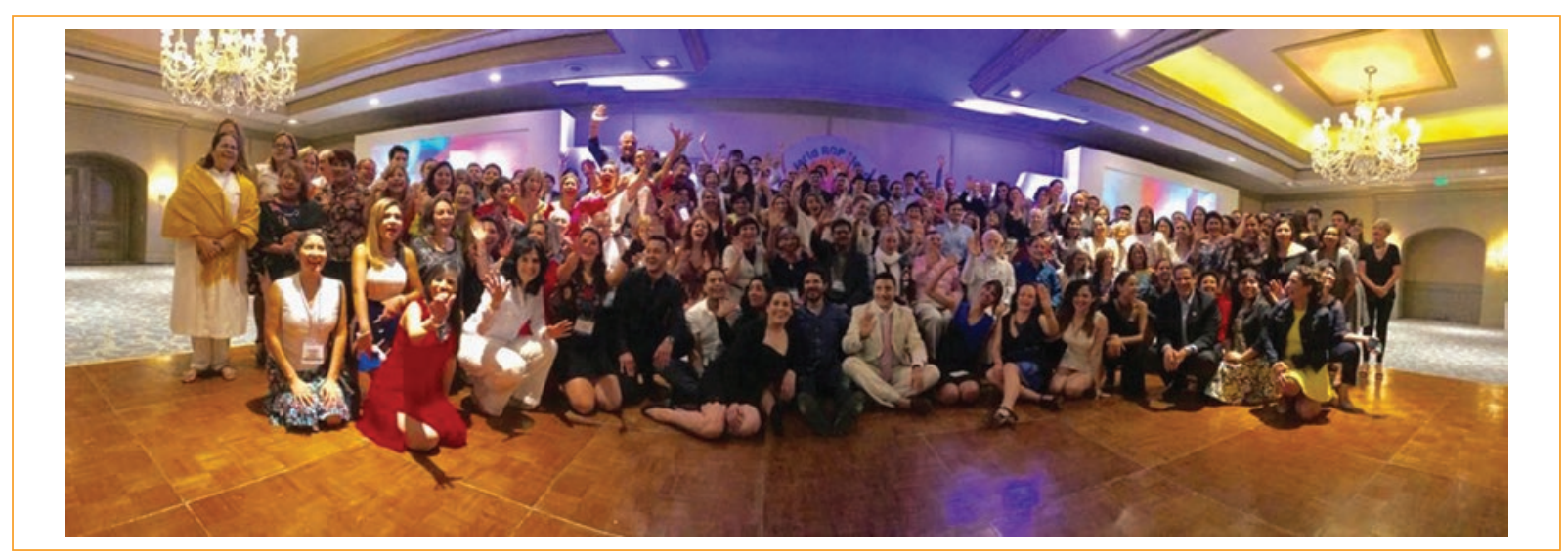

Figura 7. IV Congreso Mundial de Retinopatía del Prematuro en la ciudad de Cancún, 2017.

obligatoria la revisión a la cuarta semana de vida de la retina de todo recién nacido.

Fuimos invitados de honor el doctor Francisco Martínez Castro, expresidente de la Asociación Panamericana de Oftalmología, el Dr. Marco Antonio de la Fuente Torres, presidente de la Sociedad Mexicana de Oftalmología, el cual fue representado por Jaime Navarro, y yo, presidente de la Sociedad Panamericana de Retinopatía del Prematuro (Fig. 5).

La entrega simbólica del equipamiento a la Dra. Consuelo Zepeda por el Secretario de Salud, Dr. José Narro Robles, causó una ovación del público asistente. Directores de hospitales y unidades neonatales de todo el país, así como autoridades universitarias, se pusieron de pie como muestra de solidaridad al trabajo para que este programa fuera concretado (Fig. 6).

Otro de los logros en este tema fue en el año 2017, ya que tuvimos el privilegio de poder realizar el IV Congreso Mundial de Retinopatía del Prematuro en la ciudad de Cancún durante el mes de septiembre, en el que contamos con la participación de 42 países de 5 continentes, con 410 inscritos y 90 profesores internacionales. Se situó a México entre uno de los países de Latinoamérica que ya cuenta con un programa al presentar por parte de la Secretaría de Salud el ambicioso proyecto del Plan Nacional, del cual ya se hizo mención.

De las cosas que llamaron la atención de los participantes fue la utilidad del smartphone para documentar y realizar tamizaje de la enfermedad. Mediante el smartphone se mantiene un chat, donde más de 160 oftalmólogos están interconectados para la discusión instantánea y toma de decisiones de tratamiento de la enfermedad. Durante el congreso, se otorgó un curso práctico de la utilidad de tan valiosa herramienta y así poder compartir imágenes a nivel mundial. En el área de neonatología destacaron dos temas: prevención y tratamiento de la ROP y estrategias perinatales en su prevención, así mismo se informó de la estadística del personal de enfermería para atender esta población, con 3.9 por cada 1,000 habitantes. Finalmente durante la ceremonia de clausura del Congreso, se entregaron reconocimientos y 13 becas otorgadas por The Bernadotte Foundation for Children's Eye Care, Inc. (Fig. 7).

Con todo lo anterior queda más que claro que un grupo de especialistas pueden tomar el liderazgo para cambiar políticas nacionales en beneficio del control y tratamiento de patologías que se pueden prevenir si se realiza en forma coordinada, el Plan Nacional para la Detección y Manejo de la Retinopatía del Recién Nacido Prematuro es hoy una realidad. Sigamos trabajando en forma conjunta para disminuir el número de ciegos por esta patología y demostremos que este programa funciona.

\section{Responsabilidades éticas}

Protección de personas y animales. Los autores declaran que para esta investigación no se han realizado experimentos en seres humanos ni en animales.

Confidencialidad de los datos. Los autores declaran que han seguido los protocolos de su centro de trabajo sobre la publicación de datos de pacientes.

Derecho a la privacidad y consentimiento informado. Los autores declaran que en este estudio no aparecen datos de pacientes. 